\title{
Classical Physics and the Church-Turing Thesis
}

\author{
ANDREW CHI-CHIH YAO \\ Princeton University, Princeton, New Jersey
}

\begin{abstract}
Would physical laws permit the construction of computing machines that are capable of solving some problems much faster than the standard computational model? Recent evidence suggests that this might be the case in the quantum world. But the question is of great interest even in the realm of classical physics. In this article, we observe that there is fundamental tension between the Extended Church-Turing Thesis and the existence of numerous seemingly intractable computational problems arising from classical physics. Efforts to resolve this incompatibility could both advance our knowledge of the theory of computation, as well as serve the needs of scientific computing.
\end{abstract}

\section{Introduction}

One of the great scientific achievements in the last century was the formalization of the concept of computation. Due to the work of Church [1936], Turing [1936], and others, a standard model of computation emerged; today's digital computers can be regarded as implementations of this model. One reason for the acceptance of this model was the fact that many seemingly different formulations of the concept of computation, of which the Turing machine model was one, turned out to be essentially equivalent. The evidence for the Turing model to be the correct model seemed so convincing that a term, Church-Turing Thesis, evolved into existence over the years to express that conviction.

The Church-Turing Thesis $(C T)$ is the belief that, in the standard Turing machine model, one has found the most general concept for computability. In other words, if a function can be computed by any conceivable hardware system, then it can be computed by a Turing machine. This may not have been the belief of Church and Turing, but it has become the common interpretation of CT. With the development of computational complexity theory, which studies further refinements of the computability notion, another version of CT came into use. The Extended Church-Turing Thesis (ECT) makes the stronger assertion that the Turing machine model is also as efficient as any computing device can be. That is, if a function is computable by some hardware device in time $T(n)$ for input of size $n$, then it is

This research was supported in part by the National Science Foundation under grant number CCR9820855.

This work was completed while the author was visiting the Computer Science Department at City University of Hong Kong.

Author's address: Department of Computer Science, Princeton University, Princeton, NJ 08544, e-mail: yao@cs.princeton.edu.

Permission to make digital/hard copy of all or part of this material without fee for personal or classroom use provided that the copies are not made or distributed for profit or commercial advantage, the ACM copyright/server notice, the title of the publication, and its date appear, and notice is given that copying is by permission of the ACM, Inc. To copy otherwise, to republish, to post on servers, or to redistribute to lists requires prior specific permission and/or a fee.

(C) 2003 ACM 0004-5411/03/0100-0100 \$5.00 
computable by a Turing machine in time $(T(n))^{k}$ for some fixed $k$ (dependent on the problem).

CT, and especially ECT, have strong implications. They imply that at least in principle, to make future computers more efficient, one only needs to focus on improving the implementation technology of present-day computer designs. Although accepted by many, the validity of CT and ECT has also been called into question over the years. Among more recent work on the subject, we refer interested readers to the excellent discussions in Vergis et al. [1986], Steiglitz [1988], Penrose [1989, 1994], and Smith [1993, 1999]. While different viewpoints have been expressed, it is also clear that certain consensus has been reached in the literature regarding the nature of CT and ECT. Namely, CT and ECT are not statements about mathematics, but rather conjectured constraints on physical laws. They cannot be proved once and for all, since any physical laws may be subject to refutation one day. However, once a system of physical laws is specified (together with specifications on how input and output are handled), it then becomes a well-defined mathematical question whether these theses are valid. In fact, such questions have been examined for a number of particular systems (see Vergis et al. [1986], Steiglitz [1988], Penrose [1989, 1994], and Smith [1993, 1999]).

In this article, we wish to put some of these issues into sharper focus, and point out certain challenges and opportunities for research in this regard. We shall concentrate our discussions on the Extended Church-Turing Thesis.

\section{The Dilemma}

What is a computing device? It is rational to adopt the view that any physical system capable of providing reliable output is a potential computing device. With this in mind, one may observe that there is certain inconsistency between ECT and the status of scientific computing today. Note that, in general, a computation can be embedded in the evolution of a physical system, with the input data encoded as initial states of the system, and the output being the measured values of the observables (such as velocity, temperature, etc.) at some specified time. For example, consider a physical system governed by some set of explicit equations with variables $\mathbf{x}$. Let $g(\mathbf{x})$ be a function easily computable (in the Turing sense) from $\mathbf{x}$. Given the initial values of the variables $\mathbf{x}=\mathbf{x}(0)$ at time $t=0$, the value of $g(\mathbf{x}(t))$ at some $t>0$ can be regarded as the output of a computing device, provided that $g(\mathbf{x}(t))$ is robust with respect to slight perturbations of the initial values $\mathbf{x}(0)$.

Consider the following three statements:

(A) ECT is valid;

(B) Polynomial-time solvability is an appropriate criterion for computational feasibility;

(C) There are observables in many physical systems for which no known efficient algorithms exist.

These statements all seem sensible individually, but are inconsistent with one another. (A) implies that we should be able to use a Turing machine to compute in polynomial time $g(\mathbf{x}(t))$ with $\mathbf{x}(0)$ and $t$ as inputs. (B) then implies that one should be able to find a practical algorithm to compute $g(\mathbf{x}(t))$, which is at odds with $(\mathrm{C})$. 
The above inconsistency is not unexpected in the case of quantum systems, since it has been speculated for many years [Feynman 1982] that quantum systems cannot be efficiently simulated by standard Turing machines. The discovery of a polynomial-time quantum algorithm for factoring integers [Shor 1997] lends support to the hypothesis that quantum computers might be strictly more efficient than Turing machines.

For classical physical systems, however, this inconsistency is somewhat surprising, and seems to go against the prevailing opinions (but not all, see, e.g., Smith [1993]). Before considering some concrete physical systems, let us review a representative argument in favor of ECT for classical systems. The reasoning goes somewhat like this: If the physical system is governed by differential equations that behave mildly, then the standard numerical methods using grids can simulate the system accurately and in polynomial time. If the physical system behaves violently, then the physical variables are unpredictable and uncontrollable, in which case these variables cannot be used for computing purposes. (See, e.g., Penrose [1989] where the context is CT instead of ECT.) The weakness in the above argument we feel is that, in violent physical systems, even though the physical variables could behave unpredictably, there might be physical observables (which are functions of the physical variables) behaving robustly. We shall discuss in the next section how such possibilities might arise.

\section{The N-Body Problems}

Newton's gravitational theory for a system of $N$ point-like particles provides a natural setting for studying ECT. Assume that the particles have masses $m_{i}$, and occupy positions $\mathbf{r}_{\mathbf{i}}(t)$ at time $t$, where $1 \leq i \leq N$. Then Newton's theory gives the following system of equations for celestial mechanics: for $1 \leq i \leq N$,

$$
\frac{d^{2} \mathbf{r}_{\mathbf{i}}(t)}{d t^{2}}=G \sum_{j \neq i} m_{j} \frac{\mathbf{r}_{\mathbf{j}}(t)-\mathbf{r}_{\mathbf{i}}(t)}{\left|\mathbf{r}_{\mathbf{j}}(t)-\mathbf{r}_{\mathbf{i}}(t)\right|^{3}}
$$

where $G$ is the gravitational constant. The natural computational problem is then, given the $2 N$ initial positions $\mathbf{r}_{\mathbf{i}}(0)$ and velocities $\mathbf{r}_{\mathbf{i}}^{\prime}(0)$, and time $t>0$, compute the $N$ positions $\mathbf{r}_{\mathbf{i}}(t)$.

Although Newton's equations for celestial mechanics look simple, the behavior of the solutions (as a function of time $t$ ) can be very complex. We say that the solution runs into a singularity at time $t_{0}$, if the solution ceases to be analytic at $t=t_{0}$. For example, when two or more particles collide, a singularity naturally occurs. It was also recognized that at least theoretically there exists the possibility of other types of singularities. In 1895, Painlevé [1897] proved that, for $N=3$, the only singularities are due to collisions, and made the following conjecture:

Painlevé's Conjecture. For $N>3$, there exist noncollision singularities.

Many researchers had tried to settle this question without success until, in 1987, Xia [1992] proved that Painlevé's Conjecture is true for all $N>4$ (the case $N=4$ is still open). Subsequently, Gerver [1991] gave another proof of the result. Their proofs showed that, for some clever choice of initial conditions, the system can exchange its gravitational potential energy for kinetic energy at a geometrically 
faster rate. The speed of the particles increases so rapidly that at some finite time $t_{0}$, the particles go to infinity. An interesting historical account of this problem can be found in Diacu and Holmes [1996].

It has been suggested [Smith 1993] that such sigularities might be exploited to disprove ECT (or even CT). For any initial conditions $\beta$, define the predicate $P(\beta)$ to be 1 if it leads to a noncollision singularity, and 0 otherwise. Plausibly, a gravitational system might be constructed to decide the value of $P(\beta)$ by time $t=t_{0}$, while it could be hard to decide the value by Turing machines. This is not a rigorous refutation of ECT, since the gravitational system used needs to be infinite in size. Furthermore, the function $P$ may not be robust, and thus the input $\beta$ needs to be specified to infinite precision.

However, we can use this example to illustrate the possibility of a nontrivial computation by physical systems even if the systems may be chaotic. Assume that the set of singularities is of nonzero measure. Let $Q(\beta)$ denote the probability for $P\left(\beta^{\prime}\right)$ to be 1 , if the input $\beta^{\prime}$ is randomly chosen from a ball of some fixed radius centered at $\beta$. Then $Q(\beta)$ is a continuous function that can be probabilistically computed by a gravitational system (albeit of infinite size), even though the system may be chaotic.

It would be interesting to find a realistic example of a nontrivial computation performed by a Newtonian gravitational system. The size of the physical system should be a finite function, preferably polynomial in $N$, and there should be only finite accuracy in the measurement of particle position and velocity. It is not clear whether the singularity phenomenon mentioned above can be utilized to yield such an example.

We now turn to another example, Einstein's gravitational theory of $N$-body systems. The equations given by General Relativity are nonlinear partial differential equations, with suitable initial conditions (see, e.g., Misner et al. [1973]). Numerical computations for such systems have become very important for astrophysics studies, and many scientists have been actively working for years in pursuit of efficient algorithms for this problem. There are good approximation algorithms under special restrictions (such as when the velocities involved are slow or the gravitational field is weak), but a systematic and efficient solution to the general case is still elusive (see Damour [1990] for a review). There should be ample room in this rich theory for identifying good candidates to perform nontrivial computations.

\section{Conclusions}

Statements such as CT and ECT are important for computer science, since they reflect our assessment of how well the notion of computation is understood. In this article, we point out some intuitive conflict between ECT and the state of art in scientific computing, and suggest that it may be worthwhile to resolve this conflict. We conclude with some natural research problems along this direction.

(1) Investigating Mathematical Questions. Studying ECT gives added motivation for settling some interesting open questions in mathematics. For instance, we mentioned in the last section that a nonzero measure set of singularities would lead to a robust predicate useful for discussing ECT. It is an open problem in celestial mechanics whether the singularity set is of measure 0 . The answer is known to be positive for $N \leq 4$ [Saari 1977], but is open for $N>4$. 
(2) Applying Paradigms from Theoretical Computer Science. Traditionally, scientific computing and theory of computation are largely nonoverlapping fields. In recent years, some of the questions in scientific computing have begun to be examined from the theoretical computer science angle. To study ECT, a natural direction would be to apply the paradigms from theoretical computer science to the algorithmic questions concerning physical systems. For example, is it possible to embed an NP-complete problem into a computational problem in General Relativity? How close, at least theoretically, can this embedding be converted into a machine for solving NP-complete problems? In the opposite direction, one can try to find polynomial-time algorithms for various physical systems and thereby confirm the validity of ECT (e.g., see Vergis et al. [1986] and Smith [1993, 1999]).

Note that there is an advantage in studying simple systems such as the $N$-body systems. In more complex systems, there are sometimes hidden forces, and the successful embedding of a hard computational problem does not automatically imply the existence of a fast computing device even theoretically (see Vergis et al. [1986]).

(3) Developing New Paradigms. The formal framework of theory of computation has been developed mainly for problems that are logical and discrete in nature. The concept of polynomial-time feasibility is quite successful when applied to such computational problems. Would the study of scientific computations in the context of ECT lead to a different set of criteria? Some complexity models for dealing with computations in real numbers have been developed in recent years, using the standard Turing model as a guide (see, e.g., Blum et al. [1997]). Quite possibly, further modifications will be needed in order to fully address complexity questions in scientific computing.

\section{REFERENCES}

Blum, L., CuCKer, F., Shub, M., And Smale, S. 1997. Complexity and Real Computation. SpringerVerlag, New York.

Church, A. 1936. An unsolvable problem of elementary number theory. Amer. J. Math. 21, 345363.

DAmour, T. 1990. The problem of motion in Newtonian and Einsteinian gravity. In Three Hundred Years of Gravitation, S. W. Hawking and W. Israel, Eds. Cambridge University Press, 128-198.

Diacu, F., AND Holmes, P. 1996. Celestial Encounters: The Origins of Chaos \& Stability. Princeton University Press, Princeton, N.J.

FEYNMAN, R. P. 1982. Simulating physics with computers. Internat. J. Theor. Phys. 21, 467-488.

GERVER, J. 1991. The existence of pseudocollisions in the plane. J. Diff. Eq. 89, 1-68.

Misner, C. W., Thorne, K. S., AND WHeEler, J. A. 1973. Gravitation. Freeman.

PAINLEVÉ, P. 1897. Lecons sur la théorie analytic des equations différentielles. Hermann, Paris, France.

Penrose, R. 1989. The Emperor's New Mind. Oxford University Press, Oxford, England.

Penrose, R. 1994. Shadows of the Mind. Oxford University Press, Oxford, England.

SAARI, D. G. 1977. A global existence theorem for the four-body problem of Newtonian mechanics. $J$. Diff. Eq., 26, 80-111.

SHOR, P. 1997. Polynomial-time algorithms for prime factorization and discrete logarithms on a quantum computer. SIAM J. Comput. 26, 1484-1509.

SMITH, W. 1993. Church's thesis meets the N-body problem. Manuscript (http://www.neci.nec.com/ homepages/wds/works.html).

SMITH, W. 1999. Church's thesis meets quantum mechanics. Manuscript (http://www.neci.nec.com/ homepages/wds/works.html).

SteIGLITZ, K. 1988. Two nonstandard paradigms for computation: Analog machines and cellular automata. In Performance Limits in Communication Theory and Practice, J. K. Skwirzynski, Ed. Kluwer 
Academic Publishers, Dordrecht, The Netherlands, 173-192. (NATO Advanced Study Institutes Series E, No. 142.)

Vergis, A., Steiglitz, K., AND Dickinson, B. 1986. The complexity of analog computation. Math. Comput. Simulat. 28, 91-113.

TURING, A. M. 1936-1937. On comutable numbers, with an application to the Entscheidungsproblem. Proc. London Math. Soc., Ser. 2, 42, 230-265.

XIA, Z. 1992. The existence of noncollision singularities in the $n$-body problem. Ann. Math. 135, 3, $411-468$. 